\section{The Influence of Brass Compensator Thickness and Field Size on Neutron Contamination Spectrum in 18MV Elekta SL 75/25 Medical Linear Accelerator with and without Flattening Filter: A Monte Carlo Study}

\author{
Talebi A. S. ${ }^{1}$, Maleki M. ${ }^{2}$, Hejazi P. ${ }^{3 *}$, Jadidi M. ${ }^{4}$, Ghorbani \\ R. 5
}

\begin{abstract}
Background: One of the most significant Intensity Modulated Radiation Therapy treatment benefits is a high target to normal tissue dose ratio. To improve this advantage, an additional accessory such as a compensator is used to deliver doses. Compensator-based IMRT treatment is usually operated with an energy higher than $10 \mathrm{MV}$. Photoneutrons, which have high linear energy transfer and radiobiological effectiveness, are produced by colliding high-energy photon beams with linear accelerator structures, then they deliver the unwanted doses to patients and staff. Therefore, the neutron energy spectra should be determined in order to calculate and reduce the photoneutron risk.

Objective: We have conducted a comprehensive and precise study on the influence of brass compensator thickness and field size on neutron contamination spectrum in an Elekta SL 75/25 medical linear accelerator with and without the flattening filter by Monte Carlo method.
\end{abstract}

Material and Methods: MCNPX MC Code version 2.6.0 was utilized to simulate the detailed geometry of Elekta SL 75/25 head components based on Linac's manual. This code includes an important feature to simulate the photo-neutron interactions. Photoneutrons spectrum was calculated after the Linac output benchmarking based on tuning the primary electron beam.

Results and Conclusion: Based on the Friedman and Wilcoxon nonparametric tests results $(\mathrm{P}<0.05)$, photoneutron fluence directly depends on the field size and compensator thickness. Moreover, the unflattened beam provides lower photoneutron fluence than the flattened beam. Photoneutrons fluence is not negligible in compensator-based IMRT treatment. However, in order to optimize treatment plans, this additional and unwanted dose must be accounted for patientss.

Keywords

Photoneutron, Spectrum, MCNP, Compensator, Field Size

\section{Introduction}

$\Omega$ oday, cancer is the most common cause of death in the world [1]. More than $50 \%$ of all cancer patients receive radiation therapy [2]. One of the routine radiotherapy techniques is Intensity Modulated Radiation Therapy (IMRT). IMRT delivers high radiation doses
${ }^{1}$ M.Sc. Graduate of

Medical Physics, Department of Medical Physics,

Semnan University of

Medical Sciences, Sem-

nan, Irann

${ }^{2}$ M.Sc. Graduate of

Medical Physics, Depart-

ment of Medical Phys-

ics, Isfahan University

of Medical Sciences,

Isfahan, Iran

${ }^{3}$ Assistant Professor,

Department of Medical

Physics, Semnan Univer-

sity of Medical Sciences,

Semnan, Iran

${ }^{4}$ Associate Professor,

Department of Medical

Physics, Semnan Univer-

sity of Medical Sciences,

Semnan, Iran

${ }^{5}$ Professor, Head of

Social Determinants

of Health Research

Center, Semnan Uni-

versity of Medical

Sciences,Semnan, Iran

*Corresponding author:

P. Hejazi

Assistant Professor,

Department of Medical

Physics, Semnan Univer-

sity of Medical Sciences,

Semnan, Iran

E-mail: hejazip@se-

mums.ac

Received: 05 March 2018

Accepted: 22 April 2018 
to tumors and then provides a high target to normal tissue dose ratio [3]. In this technique for achieving a field of variable intensity, multi-leaf collimator (MLC) or compensator IS utilized. Basically, variations of compensator are based on their continual intensity, and are made of materials with different densities. In contrast with MLC, compensator-based IMRT has some advantages like simplifying quality assurance, treatment delivery and dose calculation [4]. Photon beams with energies higher than $10 \mathrm{MV}$ are used in compensatorbased IMRT. When these high energy photon beams collide with nuclei of high atomic number materials which are located along the path of the beam including linear accelerator (Linac) head structures (target, primary collimators, flattening filter and jaws), neutrons are produced by $(\gamma, n)$ and (e,e'n) interactions and contaminate therapeutic beam. The crosssection for $(\gamma, n)$ interaction is approximately 137 times higher than (e, e'n) interaction; as a result, (e, e'n) interaction generates negligibility neutrons versus $(\gamma, n)$ interaction [5]. The average threshold energy for photoneutron interactions is $8 \mathrm{MV}$. Thus, photoneutrons are generated when photon energy is higher than threshold energy [6]. According to NCRP 116 recommendation, the quality factor for neutrons with energy range $0.1-2 \mathrm{Mev}$ is 20 [7]. These particles have a high linear energy transfer (LET) and radiobiological effectiveness (RBE) delivering an unwanted dose to patients and personals which are induced secondary cancers [8]. In order to calculate and reduce the photoneutron risk, neutron energy spectra should be determined [9]. Photoneutron spectrometry is a very complex task due to the type of its interaction with matters; this is while, there are several methods to assess the neutron spectrum such as multi-sphere measurements, nuclear recoil, threshold methods, Monte Carlo (MC) calculation, etc. [10]. Nevertheless Monte Carlo calculations is an alternative method in this field of research. Various studies have been carried out for dif- ferent problems related to the neutron spectrometry. Some researchers investigated the MLC, wedge filters, flattening filter (FF) and field size effects on the neuron spectrum. In addition, other researchers reported it at different points around many types of Linac by employing the Monte Carlo method in most of their projects. Based on all publications, we can conclude that the contaminated neutron spectrum depends dominantly on the field size, the energy beam, type of Linac, treatment room geometry, the wall composition of the room and the position of the measurement point. Due to disagreements on the influence of field size on the neutron spectrum in articles and the lack of the study about compensator effects on the neutron spectrum, we have conducted a comprehensive and precise study on the influence of brass compensator thickness and field size on neutron contamination spectrum in an Elekta SL 75/25 medical linear accelerator with and without flattening filter by Monte Carlo method.

\section{Material and Methods}

\section{Linac's Head Simulation}

MCNPX MC Code version 2.6.0 was used to simulate the detailed geometry of Elekta SL 75/25 head components based on Linac's manual. This code includes an important feature that can simulate photo-neutron interactions [11]. Linac is operated by an $18 \mathrm{MV}$ photon beam. As shown in Figure 1, the main structures of Linac's head consist of target $(90 \% \mathrm{~W}$ and $10 \% \mathrm{Re})$, primary collimators $(96 \% \mathrm{~W}, 3.75 \% \mathrm{Ni}$ and $1.25 \% \mathrm{Fe})$, flattening filter (steel), ion chamber (Maylar), upper jaws $(96 \% \mathrm{~W}, 3.75 \% \mathrm{Ni}$ and $1.25 \% \mathrm{Fe})$, lower jaws $(96 \% \mathrm{~Pb}$ and $4 \% \mathrm{Sn})$, and shielding $(96 \% \mathrm{~W}$, $3.75 \% \mathrm{Ni}$ and $1.25 \% \mathrm{Fe}$ ). In order to tune the simulated Linac, the percentage depth dose (PDD) and beam profile data derived from MCNP calculation should match the experimental dose data in a water phantom within $1 \%$. This Linac was validated in our previous 

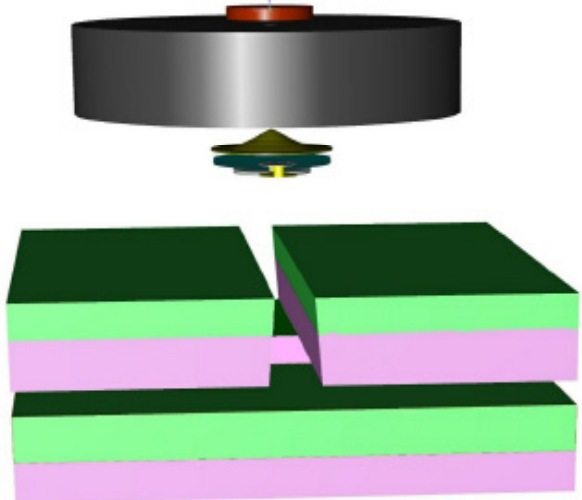

Figure 1: The Geometry of Linac's Head

study [12]. To determine the percentage depth dose and beam profile data in the source-surface distance (SSD) of $100 \mathrm{~cm}$, a water phantom with dimensions of $60 \times 60 \times 50 \mathrm{~cm}^{3}$ was simulated. To achieve differences less than $1 \%$ between measurement and calculation data, the characteristics of incident primary electron beam such as mean energy, radial distribution and energy distribution were adjusted [13]. The cut-off energy for electron and photon were selected as $10 \mathrm{Kev}$ and $700 \mathrm{Kev}$, respectively. Also, to calculate dose data *F8 tally was used. A water phantom (RFA-300) and a Farmer ionization chamber (FC65-P, volume $=65 \mathrm{~cm}^{3}$ ) were utilized to gather experimental data. The number of primary electrons was selected to be $1 \times 10^{9}$.

\section{Calculation of Photoneutron Spec- trum}

Photoneutron spectrum was calculated after Linac output benchmarking according to tuning the primary electron beam. To calculate the photoneutron spectrum, the code was slightly modified. The photoneutron production does not occur lower than $7 \mathrm{MeV}$. Therefore, the cut-off energy for electron and photon was altered to 7Mev. The fourth entry of PHYS: P card is ispn that controls photonuclear particle production. This entry must be chosen as -1 for an analogue photonuclear production. To carry out this calculation, a void sphere cell with a dimension of $10 \mathrm{~cm}$ diameter was modelled in $\mathrm{SSD}=100$ from the target. The photoneutrons crossing the void cell were accounted by F4 tally, which is used for the determination of flux average over a cell. The geometry of the brass compensator as a cylinder with $21 \mathrm{~cm}$ diameter and a variable height $(0.5,1,2,3,6$ and $8 \mathrm{~cm}$ ), was defined in $67.2 \mathrm{~cm}$ from the target. The brass compensator compositions are $3 \% \mathrm{~Pb}, 61.5 \% \mathrm{Cu}$ and $35.5 \% \mathrm{Zn}$ with the density of $8.22 \mathrm{gr} / \mathrm{cm}^{3}$. Photoneutron spectrum was investigated and calculated for the following cases: (a for flattened and unflattened 18MV photon beam in different field sizes $(5 \times 5$, $10 \times 10,15 \times 15$ and $20 \times 20$ ) and b) in the presence of compensators with thickness $0.5,1,2$, 3,6 and $8 \mathrm{~cm}$ for different field sizes in flattened and unflattened 18-MV photon beam. To correctly compare the photoneutron spectrum in different cases, the neutron spectrum was obtained for the same absorbed dose in an isocenter. The uncertainty for photoneutron spectrum and dose calculation was less than $2 \%$ and $1 \%$, respectively.

\section{Results}

\section{Linac Validation}

Linac validation was conducted by comparing $\mathrm{MC}$ calculation and practical measurement data for the open field. Figure 2 shows that the difference between two PDD curves resulting from $\mathrm{MC}$ and empirical measurement is within $1 \%$ for the depths after $3 \mathrm{~cm}$ (build-up depth). Difference less than $2 \%$ is seen for the points located in the flat region in beam profiles (Figure 3). Our validation results are in agreement with other studies and are in accordance with our previous study [12]. Finally, we have chosen the features of incident primary electron beam based on the validation results (mean energy $=17.8 \mathrm{MV}$, radial distribution $=0.35 \mathrm{~cm}$ and Gaussian energy distribution with FWHM=7\%). 


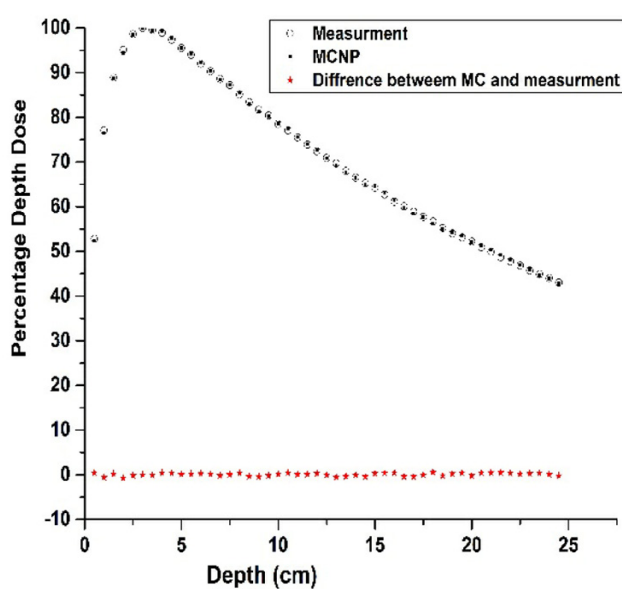

Figure 2: Percentage depth dose curves difference between empirical measurements and $\mathrm{MC}$ calculation for $10 \times 10 \mathrm{~cm}^{2}$ field size

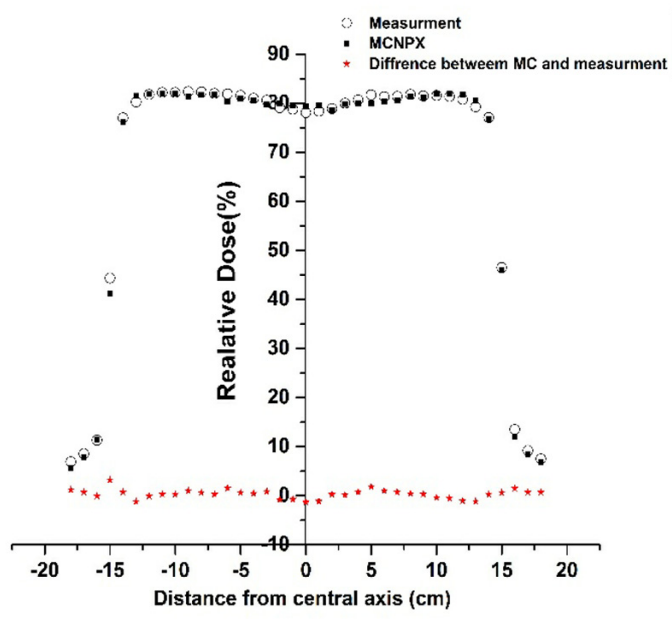

Figure 3: Beam profiles difference between empirical measurements and MC calculation for $30 \times 30 \mathrm{~cm}^{2}$ field size.

\section{Photoneutrons spectrum}

The results of MC calculation to observe the effect of flattening filter on photoneutron spectrum are shown in Figure 4. Based on Wilcoxon's nonparametric test results $(\mathrm{P}<0.05)$, Photoneutrons fluence reduced with removing FF. Photoneutron fluence was $72.8 \%, 71.7 \%$,
$70.4 \%, 70.2 \%, 69.5 \%, 68.8 \%$ and $64.6 \%$ lower for unflattened beams in $10 \times 10 \mathrm{~cm}^{2}$ field size for te open field and compensator field with $0.5,1,2,3,6$ and $8 \mathrm{~cm}$ thickness, respectively. The photoneutron fluence is listed in Table 1 for the unflattened and flattened beams in different field sizes and different thicknesses of the Brass compensator. We performed Friedman's nonparametric test to assess the effect of field size on photoneutron spectrum, and found that photoneutron fluence increased when field size increased for all simulated cases $(\mathrm{P}<0.05)$. The ratio of photoneutron fluence for $20 \times 20 \mathrm{~cm}^{2}$ field size to photoneutron fluence for $5 \times 5 \mathrm{~cm}^{2}$ field size is approximately 1.3 for the flattened beam in open field and compensator field with different thicknesses. This proportion is approximately 1.2 for the unflattened beam. As the results of Friedman test $(\mathrm{P}<0.05)$ show, it can be concluded that there is a significant difference among photoneutron fluences in different thicknesses of the compensator. Figures 5 and 6 reveal that photoneutron fluence increased by the increment compensator thickness and this effect is the same for the unflattened and flattened beams. As a case in point, when the compensator thickness increases from $0.5 \mathrm{~cm}$ to $8 \mathrm{~cm}$, the photoneutron fluence increases 4.5 times for flattened beam in $5 \times 5 \mathrm{~cm}^{2}, 10 \times 10 \mathrm{~cm}^{2}$, $15 \times 15 \mathrm{~cm}^{2}$ and $20 \times 20 \mathrm{~cm}^{2}$ field sizes. This variation is 5.5 times for the unflattened beam.

\section{Discussion}

\section{The effect of FF on Photoneutron Spectrum}

According to our results, by removing FF, the shape of photoneutron spectrum does not considerably change, while the photoneutron fluence decreases notably. The main reason for this variation is that by removing FF photons, fluence increases in isocenter due to the lack of flattening filter attenuation effect. Therefore, to achieve the same dose in isocenter for unflattened and flattened beams, photon flu- 

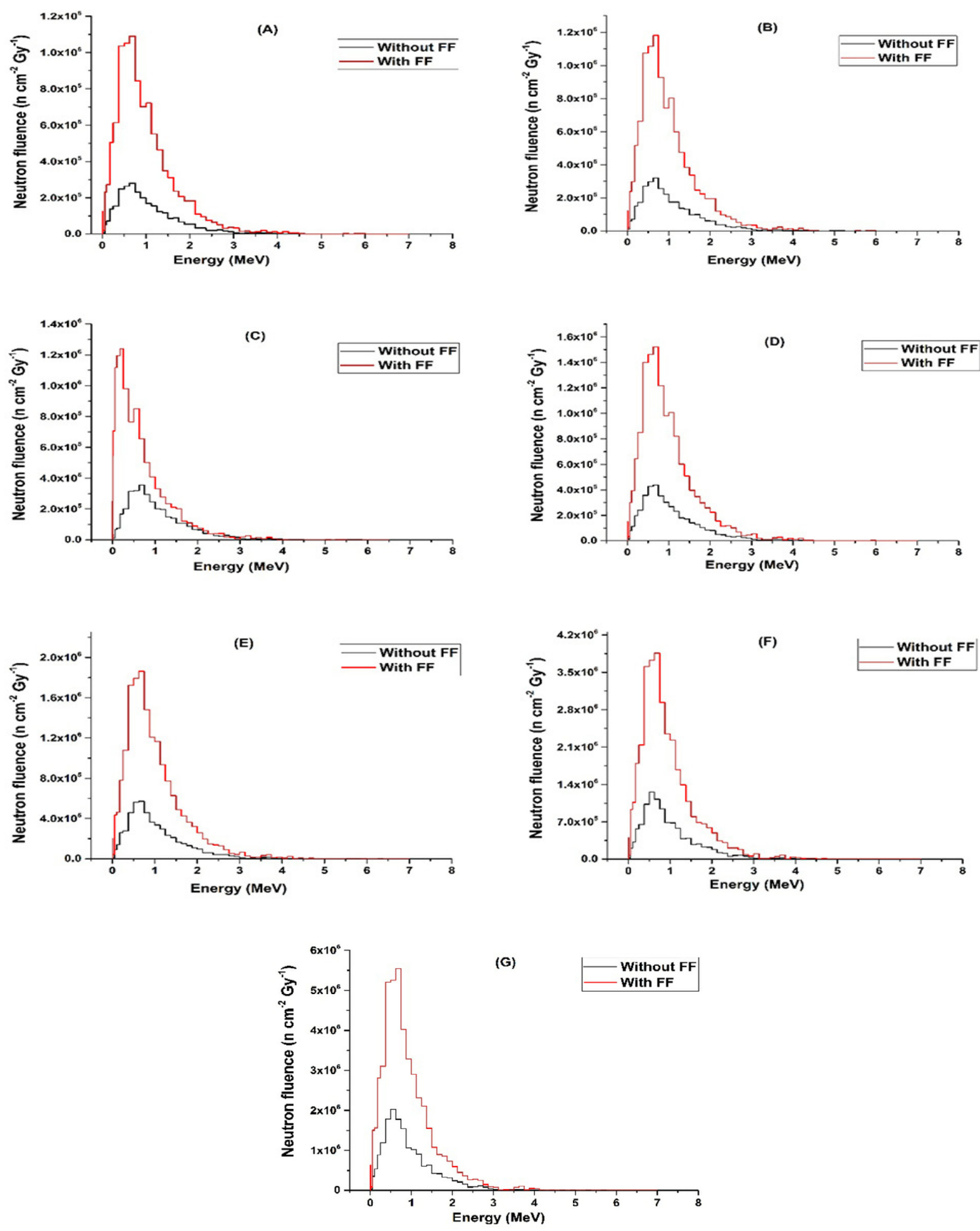

Figure 4: Compression of photoneutron spectrum for the unflattened and flattened beams in $10 \times 10 \mathrm{~cm}^{2}$ field size. A) Without compensator. B) Compensator with $0.5 \mathrm{~cm}$ thickness. C) Compensator with $1 \mathrm{~cm}$ thickness. D) Compensator with $2 \mathrm{~cm}$ thickness. E) Compensator with $3 \mathrm{~cm}$ thickness. F) Compensator with $6 \mathrm{~cm}$ thickness. G) Compensator with $8 \mathrm{~cm}$ thickness. 
Table 1: Photoneutron fluence.

\begin{tabular}{|c|c|c|c|}
\hline \multirow{2}{*}{$\begin{array}{l}\text { The thick- } \\
\text { ness of } \\
\text { compensa- } \\
\text { tor }(\mathrm{cm})\end{array}$} & \multirow{2}{*}{$\begin{array}{l}\text { Field } \\
\text { size } \\
\left(\mathrm{cm}^{2}\right)\end{array}$} & \multicolumn{2}{|c|}{$\begin{array}{l}\text { Photoneutron flu- } \\
\text { ence }\left(\left(\mathrm{n} / \mathrm{cm}^{2} \mathrm{~Gy}-((\mathrm{n} /\right.\right. \\
\left.\left.\mathrm{cm}^{2} \mathrm{~Gy}^{-1}\right) \times 10^{6}\right)\end{array}$} \\
\hline & & $\begin{array}{c}\text { flattened } \\
\text { beam }\end{array}$ & $\begin{array}{c}\text { unflattened } \\
\text { beam }\end{array}$ \\
\hline \multirow{4}{*}{0} & $5 \times 5$ & $8.79 \pm 0.241$ & $2.52 \pm 0.067$ \\
\hline & $10 \times 10$ & $10.3 \pm 0.275$ & $2.80 \pm 0.071$ \\
\hline & $15 \times 15$ & $10.9 \pm 0.288$ & $2.94 \pm 0.065$ \\
\hline & $20 \times 20$ & $11.6 \pm 0.299$ & $3.02 \pm 0.075$ \\
\hline \multirow{4}{*}{0.5} & $5 \times 5$ & $9.82 \pm 0.269$ & $2.78 \pm 0.094$ \\
\hline & $10 \times 10$ & $11 \pm 0.271$ & $3.11 \pm 0.071$ \\
\hline & $15 \times 15$ & $12.4 \pm 0.275$ & $3.21 \pm 0.820$ \\
\hline & $20 \times 20$ & $12.9 \pm 0.272$ & $3.53 \pm 0.072$ \\
\hline \multirow{4}{*}{1} & $5 \times 5$ & $10.7 \pm 0.284$ & $3.07 \pm 0.082$ \\
\hline & $10 \times 10$ & $11.7 \pm 0.295$ & $3.46 \pm 0.080$ \\
\hline & $15 \times 15$ & $12.5 \pm 0.293$ & $3.5 \pm 0.083$ \\
\hline & $20 \times 20$ & $13.3 \pm 0.297$ & $3.66 \pm 0.084$ \\
\hline \multirow{4}{*}{2} & $5 \times 5$ & $12.9 \pm 0.385$ & $3.8 \pm 0.102$ \\
\hline & $10 \times 10$ & $14.3 \pm 0.375$ & $4.26 \pm 0.100$ \\
\hline & $15 \times 15$ & $15.5 \pm 0.413$ & $4.41 \pm 0.106$ \\
\hline & $20 \times 20$ & $16.6 \pm 0.432$ & $4.57 \pm 0.111$ \\
\hline \multirow{4}{*}{3} & $5 \times 5$ & $15.7 \pm 0.252$ & $4.88 \pm 0.127$ \\
\hline & $10 \times 10$ & $17.5 \pm 0.280$ & $5.34 \pm 0.130$ \\
\hline & $15 \times 15$ & $19 \pm 0.294$ & $5.7 \pm 0.133$ \\
\hline & $20 \times 20$ & $20.6 \pm 0.532$ & $6.09 \pm 0.125$ \\
\hline \multirow{4}{*}{6} & $5 \times 5$ & $30.5 \pm 0.879$ & $9.52 \pm 0.262$ \\
\hline & $10 \times 10$ & $34.3 \pm 0.993$ & $10.7 \pm 0.240$ \\
\hline & $15 \times 15$ & $38.19 \pm 0.109$ & $11.6 \pm 251$ \\
\hline & $20 \times 20$ & $42.6 \pm 0.116$ & $12.2 \pm 0.356$ \\
\hline \multirow{4}{*}{8} & $5 \times 5$ & $43.3 \pm 0.522$ & $15.2 \pm 0.433$ \\
\hline & $10 \times 10$ & $48.3 \pm 0.280$ & $17.1 \pm 0.400$ \\
\hline & $15 \times 15$ & $53.1 \pm 0.294$ & $18.5 \pm 0.440$ \\
\hline & $20 \times 20$ & $58.3 \pm 0.302$ & $19.1 \pm 0.421$ \\
\hline
\end{tabular}

ence production decreases in the target for the unflattened beam. A main result of this study was that photoneutron interactions decreased in Linac's head structures which are made of high atomic number. Although our results are in agreement with the related publications [14, 15], different reasons were mentioned about the photoneutron fluence reduction in the unflattened beam. One of these reasons is the flattening filter capability in photoneutron production [15]. As mentioned above, FF compositions are made of low-atomic-number materials in Elekta SL 75/2 Linac. Accordingly, due to their low photoneutron cross-section, the contribution of flattening filter in photoneutron production is negligible and is inconsistent with the results of the present study.

\section{The effect of Field Size on Photo- neutron Spectrum}

As presented in Table 1, the photoneutron fluence decreases with a decrease in the field size in the open field, and the compensator field for with and without FF present in Linac's head; these variations can be explained by the contribution of secondary collimators in photoneutron attenuation. When field size decreases, the secondary collimators will be closed. Therefore, photoneutrons originated from the upper structures are attenuated by secondary collimators because of its thickness. In addition, the photoneutrons generated by secondary collimators will be stopped locally. Most probably, the secondary collimators have a significant attenuated effect. By increasing field size, the interval between secondary collimators increases. As a result, more photoneutrons will be received by the detector in isocenter without the attenuation effect of the secondary collimators. Additionally, the photoneutron fluence increases with the field size in the presence of Brass compensator with different thicknesses $(0.5,1,2,3,6$ and $8 \mathrm{~cm})$. Based on the compensator compositions ( $3 \%$ $\mathrm{Pb}, 61.5 \% \mathrm{Cu}$ and $35.5 \% \mathrm{Zn}$ ), as field size enlarges, a larger surface of the compensator is irradiated by high energy photons. Then, the photoneutron fluence increases by increasing the number of photon-neutron interactions in the compensator. Many authors have investigated the dependency of field size on the photoneutron spectrum [16-22]. Nevertheless, this investigation has become a controversial issue. 

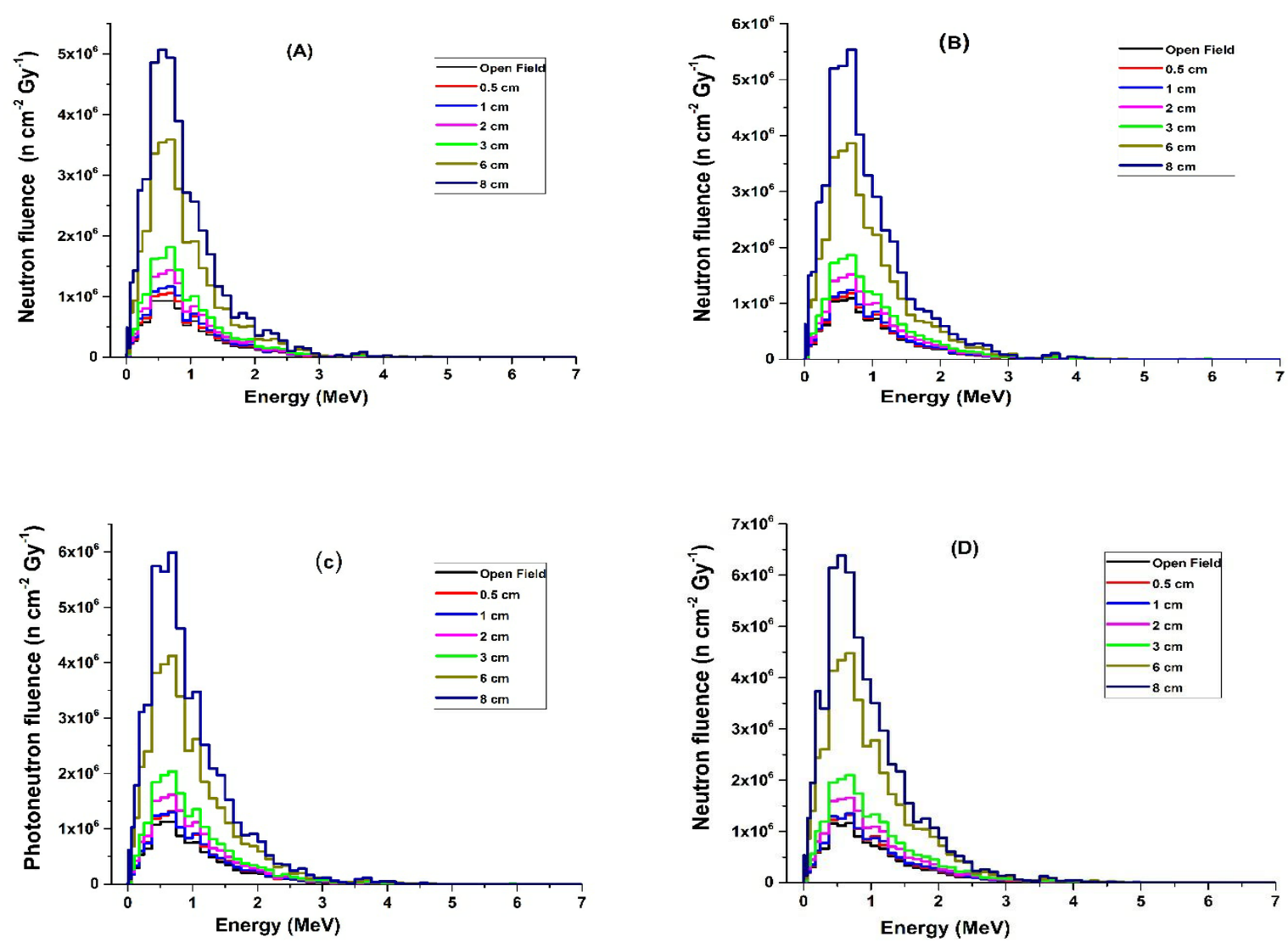

Figure 5: . Compression of photoneutron spectrum for different compensator thicknesses for flattened beam. A) $5 \times 5 \mathrm{~cm}^{2}$ field size B) $10 \times 10 \mathrm{~cm}^{2}$ field size C) $15 \times 15 \mathrm{~cm}^{2}$ field size D) $20 \times 20$ $\mathrm{cm}^{2}$ field.

Our results are completely in agreement with several publications $[16,17,19]$, but disagree with MC and experimental studies by several researchers [20-22]. It seems that high statistical uncertainty of publications has influenced the results. As mentioned previously, our MC calculation is performed with a statistical uncertainty less than $2 \%$.

\section{the effect of Compensator Thick- ness on Photoneutron Spectrum}

The photoneutron spectrum shape does not change significantly with the increase in compensator thickness. Our results exhibited that photoneutron fluence increases with compensator thickness in the flattened and unflattened beams. The increase in the photoneutron flu- ence with compensator thickness can be explained by the fact that photon beams are attenuated when the compensator is located in the beam path. Subsequently, the absorbed dose decreases in the isocenter. In order to achieve the same absorbed dose, the photon fluence should be increased. Hence, by an increase in the photon fluence with compensator thickness, the number of photoneutron interactions and, in turn, photoneutron fluence will increase. Recent investigations conducted on the aspects of IMRT treatment show an increasing neutron contamination in comparison with conventional treatments [23, 24].

\section{Conclusion}

In this study, the photoneutron spectrum was 

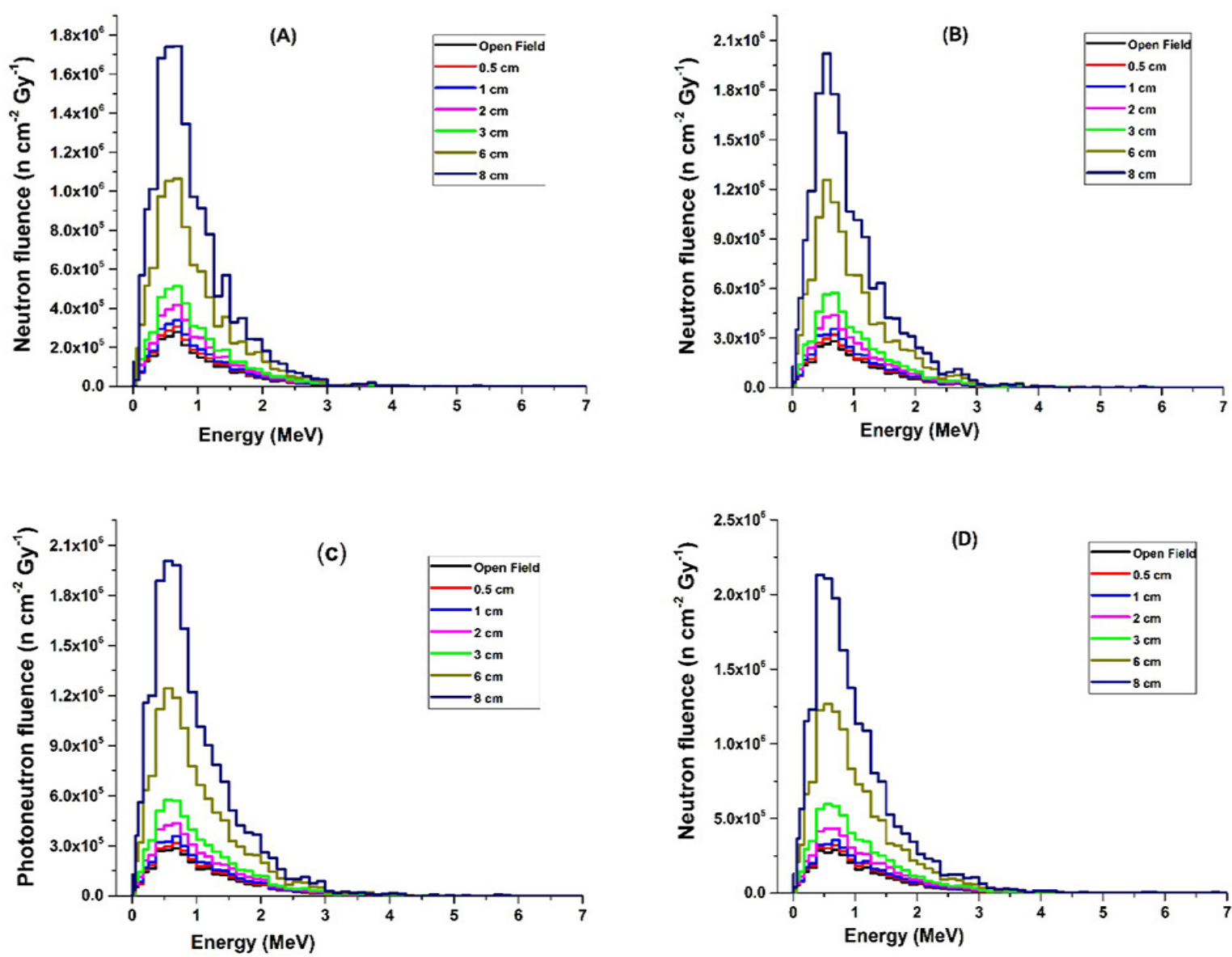

Figure 6: Compression of photoneutron spectrum for different compensator thicknesses for unflattened beam. A) $5 \times 5 \mathrm{~cm}^{2}$ field size B) $10 \times 10 \mathrm{~cm}^{2}$ field size C) $15 \times 15 \mathrm{~cm}^{2}$ field size D) $20 \times 20$ $\mathrm{cm}^{2}$ field size

calculated through MC method by mounting different thicknesses of brass compensator on the head of ELEKTA SL75/25 Linac. Although experimental methods are usually associated with uncertainties, MC method presents accurate calculations. The application of compensator and FF leads to enhanced photoneutron fluence and increased field size. The radioprotection recommendations indicate harmful biological effects of contaminated neutrons on patients and personnel. A careful study of this work can improve the benefit-risk ratio of IMRT-based compensator treatment by photoneutron spectrum determination.

\section{Acknowledgment}

This study is an M.Sc thesis in the medical physics. The authors are grateful from Semnan University of Medical Sciences for financial support of this work.

\section{Conflict of Interest}

None

\section{References}

1. Torre LA, Sauer AMG, Chen MS, KagawaSinger M, Jemal A, Siegel RL. Cancer statistics for Asian Americans, Native Hawaiians, and Pacific Islanders, 2016: Converging incidence in males and females. CA Cancer $J$ 
Clin. 2016;66:182-202.

2. Ron E. Ionizing radiation and cancer risk: evidence from epidemiology. Radiat Res. 1998;150:S30-S41.

3. Bortfeld T. IMRT: a review and preview. Phys Med Biol. 2006;51:R363.

4. Chang SX, Cullip TJ, Deschesne KM, Miller EP, Rosenman JG. Compensators: An alternative IMRT delivery technique. J App/ Clin Med Phys. 2004;5:15-36.

5. Vega-Carrillo HR, Hernández-Almaraz B, Hernández-Dávila VM, Ortíz-Hernández A. Neutron spectrum and doses in a $18 \mathrm{MV}$ LINAC. Journal of radioanalytical and nuclear chemistry. 2010;283:261-5.

6. Swanson WP. Calculation of neutron yields released by electrons incident on selected materials. Health Phys. 1978;35:353-67. PubMed PMID: 701032.

7. Marx MV. NCRP Report No. 116 Limitation of Exposure to lonizing Radiation. J Vasc Interv Radiol. 1995;6:96.

8. Guo S, Ziemer PL. Health physics aspects of neutron activated components in a linear accelerator. Health Phys. 2004;86:S94-S102.

9. Schneider U, Lomax A, Timmermann B. Second cancers in children treated with modern radiotherapy techniques. Radiother Oncol. 2008;89:135-40. doi: 10.1016/j. radonc.2008.07.017. PubMed PMID: 18707783.

10. Brooks F, Klein H. Neutron spectrometry-historical review and present status. Nuclear Instruments and Methods in Physics Research Section A: Accelerators, Spectrometers, Detectors and Associated Equipment. 2002;476:1-11.

11. Forster R, Godfrey T. MCNP-a general Monte Carlo code for neutron and photon transport. Monte-Carlo Methods and Applications in Neutronics, Photonics and Statistical Physics: Springer; 1985. p. 33-55.

12. Talebi AS, Hejazi P, Jadidi M, Ghorbani R. A Monte Carlo study on Photoneutron Spectrum around Elekta SL75/25 18 MV linear accelerator. International Journal of $A d$ vanced Biological and Biomedical Research. 2016;4:48-57.
13. Sheikh-Bagheri D. Monte Carlo study of photon beams from medical linear accelerators: optimization, benchmark and spectra: Carleton University Ottawa, Canada; 1998.

14. Mesbahi A. A Monte Carlo study on neutron and electron contamination of an unflattened 18-MV photon beam. Appl Radiat Isot. 2009;67:55-60.

15. Kry SF, Titt U, Pönisch F, Vassiliev ON, Salehpour M, Gillin M, et al. Reduced Neutron Production Through Use of a FlatteningFilter-Free Accelerator. International Journal of Radiation Oncology Biology Physics. 2007;68:1260-4.

16. Biltekin F, Yeginer M, Ozyigit G. Investigating in-field and out-of-field neutron contamination in high-energy medical linear accelerators based on the treatment factors of field size, depth, beam modifiers, and beam type. Physica Medica: European Journal of Medical Physics. 2015;31:517-23.

17. Al-Ghamdi $\mathrm{H}$, Al-Jarallah M, Maalej N. Photoneutron intensity variation with field size around radiotherapy linear accelerator 18-MeV X-ray beam. Radiation Measurements. 2008;43:S495-S9.

18. Chibani 0, Ma CMC. Photonuclear dose calculations for high-energy photon beams from Siemens and Varian linacs. Med Phys. 2003;30:1990-2000.

19. Garnica-Garza H. Characteristics of the photoneutron contamination present in a high-energy radiotherapy treatment room. Phys Med Biol. 2005;50:531.

20. Mesbahi A, Keshtkar A, Mohammadi E, Mohammadzadeh M. Effect of wedge filter and field size on photoneutron dose equivalent for an 18 MV photon beam of a medical linear accelerator. Appl Radiat Isot. 2010;68:84-9.

21. Kim HS, Park YH, Koo BC, Kwon JW, Lee JS, Choi HS. Evaluation of the photoneutron field produced in a medical linear accelerator. Radiat Prot Dosimetry. 2007;123:323-8. doi: 10.1093/rpd/ncl162. PubMed PMID: 17077093.

22. Mao X, Kase K, Kleck J, Liu J, Johnsen S, Nelson W. Neutron sources in the Varian Clinac 2100C/2300C medical accel- 
erator calculated by the EGS4 code. SCAN9702021. Health Physics, 1996;72:524-9.

23. Ahmed S, Rafi M, Jamil R, Khan MF, Maqbool A, Hashmi A. Neutron contamination in conventional 3DCRT treatment and step and shoot IMRT technique using $10 \mathrm{mv}$ photon beam. Physica Medica: European
Journal of Medical Physics. 2016;32:321.

24. Howell RM, Ferenci MS, Hertel NE, Fullerton GD. Investigation of secondary neutron dose for $18 \mathrm{MV}$ dynamic MLC IMRT delivery. Med Phys. 2005;32:786-93. doi: 10.1118/1.1861162. PubMed PMID: 15839351. 